\title{
Hearing Status of Children and Adolescents With Familial Adenomatous Polyposis
}

\author{
URSULA M. FINDLEN ${ }^{1,2}$, REBECCA L. BELT ${ }^{1}$, LING FAN $^{3}$, \\ NIKIA BRIDGES ${ }^{1}$, JASON A. BENEDICT ${ }^{4}$ and STEVEN H. ERDMAN ${ }^{3,5}$ \\ Departments of ${ }^{1}$ Audiology and ${ }^{5}$ Pediatrics, ${ }^{3}$ Division of Gastroenterology, Hepatology and Nutrition, \\ Nationwide Children's Hospital and The Ohio State University College of Medicine, Columbus, OH, U.S.A.; \\ ${ }^{2}$ Department of Otolaryngology, Head and Neck Surgery, \\ The Ohio State University College of Medicine, Columbus, OH, U.S.A.; \\ ${ }^{4}$ Center for Biostatistics, Department of Biomedical Informatics, \\ The Ohio State University College of Medicine, Columbus, OH, U.S.A.
}

\begin{abstract}
Background/Aim: Chemopreventative therapeutics may be helpful in familial adenomatous polyposis (FAP) management; however, prospective chemopreventative studies are complicated by potential ototoxicity and pre-existing hearing loss. The aim of this study was to establish and compare baseline hearing status of children and adolescents with FAP and their unaffected siblings. Patients and Methods: Twenty FAP pediatric patients with documented mutation of the adenomatous polyposis coli (APC) gene and nine unaffected sibling controls underwent baseline hearing evaluation, including audiometry, speech perception testing, and middle and inner ear physiologic measures. Results of the FAP cohort were compared to the unaffected sibling cohort. Results: Two (5\%) children with FAP presented with baseline hearing loss of unknown etiology, likely unrelated to their FAP diagnosis. No significant differences were found in any of the hearing measures between groups. Conclusion: Mutation of the APC gene is not necessarily indicative of higher risk for baseline hearing loss in the pediatric population.
\end{abstract}

Familial adenomatous polyposis (FAP) is a life-long disease caused by an inherited or acquired mutation in the adenomatous polyposis coli $(A P C)$ gene, which results in the

This article is freely accessible online.

Correspondence to: Ursula M. Findlen (ORCID ID: 0000-00018971-0700), Ph.D., Division of Clinical Therapies- Audiology Department, Nationwide Children's Hospital, 700 Children's Drive, Columbus, OH 43205, U.S.A. Tel: +1 6147226526, Fax: +1 6147223904,e-mail: ursula.findlen@nationwidechildrens.org

Key Words: Familial adenomatous polyposis, hearing loss, ototoxicity, chemoprevention. initiation of adenoma growth in the gastrointestinal tract (1). The development of colorectal cancer (CRC) in individuals carrying a mutation in $A P C$ is nearly inevitable and typically occurs in the third to fourth decade of life, much earlier than the development of sporadic CRC in the general population. Prophylactic surgery with removal of the colon is viewed as standard of care for patients with FAP with consequences that adversely affect quality of life (1). Timing of surgery is multifactorial and influenced by both medical and psychosocial factors. The slowing of adenoma progression through pharmacologic intervention would allow deferment of colectomy into mid-adulthood and could possibly impact the extra-intestinal manifestations of FAP. The possibility of both FAP-associated hearing loss and the ototoxic nature of specific chemopreventative agents have overshadowed the development of these therapeutic agents.

The $A P C$ gene is considered essential for hearing as it plays a role in cytoskeletal microtubule development in various cells of the cochlea and olivocochlear neurons (2). Animal models possessing mutant $A P C$ have shown reduced auditory function, as measured by auditory brainstem response (ABR) testing (3). Previous studies have investigated the occurrence of hearing loss in adults with FAP and found some differences in hearing thresholds when compared to age normative data $(4,5)$. Jones et al. (4) reported statistically significant differences in hearing thresholds at $500 \mathrm{~Hz}$ and $1000 \mathrm{~Hz}$, while Lutz et al. (5) reported that $53.8 \%$ of young adults aged 18-25 years with FAP presented with reduced hearing thresholds. Both of these studies used normative values for hearing thresholds derived from the International Standards Organization 7029 'Acoustics- Statistical distribution of hearing thresholds as a function of age' to which to compare their sample as opposed to including a control cohort for evaluating differences in thresholds. Jones et al. (4) reported statistically significant differences in thresholds although the 
degrees of difference were not clinically significant. Therefore, questions remain as to whether true differences in hearing occur in the FAP population and whether they are clinically significant. Currently, there are no data in the medical literature to address the following questions: does mutation of the $A P C$ gene convey a hearing loss and at what age can it be detected?

Prior chemopreventative trials in FAP have focused on the use of nonsteroidal anti-inflammatory drugs (NSAIDS), such as sulindac or celecoxib, with mixed results $(6,7)$. Recent trials, both animal and human, suggest that combination therapy with an NSAID and difluoromethylornithine (DFMO) or eflornithine may have potent synergistic effects on adenoma development and regression in adults with FAP at risk for recurrent adenoma formation as well as in FAP animal models $(8,9)$. Although some clinical studies regarding the safety of DFMO in adults have indicated little or no change in hearing thresholds $(8,10,11)$, other studies in both the animal model and in adults with melanoma have suggested that DFMO may have dose-dependent ototoxic effects on hearing (12-14).

Plans for a chemoprevention trial using sulindac and DFMO in the pediatric FAP population are currently under consideration. However, before a phase I pediatric Sulindac/DFMO chemoprevention trial can be considered for children and adolescents, the pre-treatment hearing status of the study population needs to be more carefully evaluated in a systematic fashion. This is especially important given the documented propensity of younger FAP adults to present with pre-existing hearing loss (5). The specific aim of the current study is to establish the baseline hearing status of children and adolescents with mutation of the APC gene and compare this to a group of genotype negative/unaffected siblings.

\section{Patients and Methods}

Participants. This research was completed with Institutional Review Board approval. Potential genotype positive subjects were recruited through the clinical polyposis registry of Nationwide Children's hospital. A screening health questionnaire was administered to assess patient eligibility. Genotype data as well as socioeconomic status indicators, such as maternal education and family income, were gathered through reviewing patient medical charts. Inclusion criteria included children between the ages of 6 and 19 years who had documented mutation in the APC gene but were otherwise healthy and their siblings. Exclusion criteria included children with known hearing loss with known etiology, abnormality of the external ear canal or tympanic membrane, patients with acute illness that may compromise hearing and patients with established autoimmune disease. Four patients were excluded from the study due to known hearing loss due to ototoxicity from previous chemotherapy. Patients who met study criteria were briefed on the study procedures and consented/assented prior to data collection. A total of 29 children were enrolled in the study and completed the hearing evaluation: 20 FAP children/adolescents [13 male, average
Table I. Subject demographics including sex, sibling match, FAP categorization (Class: classic FAP, Att: attenuated, Sev: severe), and FAP genotype.

\begin{tabular}{|c|c|c|c|c|}
\hline FAP PID & $\begin{array}{c}\text { Age } \\
\text { (years) }\end{array}$ & Gender & $\begin{array}{c}\text { FAP } \\
\text { category }\end{array}$ & Genotype \\
\hline E-001 & 16.4 & $\mathrm{M}$ & Class & c. $3283 \mathrm{C}>\mathrm{T}$ \\
\hline E-002 & 11.0 & $\mathrm{~F}$ & Class & c. $3202 \mathrm{del} 4$ \\
\hline E-005 & 10.4 & $\mathrm{M}$ & Class & p.s117x \\
\hline E-012 & 17.8 & $\mathrm{~F}$ & Sev & c. $3810 \mathrm{~T}>\mathrm{A}$ \\
\hline E-013 & 13.2 & M & Class & c. $1866 \mathrm{C}>\mathrm{A}$ \\
\hline E-015 & 9.9 & M & Att & c. $509 \mathrm{del} 4$ \\
\hline E-018 & 8.2 & $\mathrm{~F}$ & Class & c. $2793 \mathrm{del} 4$ \\
\hline E-019 & 17.9 & M & Class & c.1759delA \\
\hline E-021 & 9.3 & M & Sev & c. $3957 \mathrm{del}$ \\
\hline E-023 & 8.5 & $\mathrm{M}$ & Class & c. $690 \mathrm{C}>\mathrm{T}$ \\
\hline E-024 & 15.4 & $\mathrm{~F}$ & Class & del exons 3-4 \\
\hline E-025 & 14.6 & M & Class & del exons 3-4 \\
\hline E-026 & 11.6 & M & Class & del exon 15 \\
\hline E-027 & 18.0 & M & Class & c. $4638-4642 \mathrm{del}$ TGAA \\
\hline E-028 & 16.9 & $\mathrm{M}$ & Att & c.5934-5937del AAAC \\
\hline E-030 & 16.1 & $\mathrm{~F}$ & Att & c.5936-5939del ACAA \\
\hline E-031 & 17.0 & $\mathrm{M}$ & Att & c.5936-5939del \\
\hline E-032 & 10.7 & $\mathrm{~F}$ & Class & c. $3203 \mathrm{C}>\mathrm{A}$ \\
\hline E-033 & 6.1 & $\mathrm{~F}$ & $\mathrm{Sev}$ & c. $3982 \mathrm{C}>\mathrm{T}$ \\
\hline E-034 & 7.3 & $\mathrm{M}$ & Sev & c. $3982 \mathrm{C}>\mathrm{T}$ \\
\hline $\begin{array}{l}\text { Non-FAP } \\
\text { PID }\end{array}$ & $\begin{array}{c}\text { Age } \\
\text { (years) }\end{array}$ & Gender & $\begin{array}{l}\text { Sibling } \\
\text { to }\end{array}$ & Genotype \\
\hline E-004 & 14.9 & $\mathrm{M}$ & E-002 & NA \\
\hline E-003 & 12.2 & $\mathrm{~F}$ & E-002 & NA \\
\hline E-006 & 6.7 & M & E-005 & NA \\
\hline E-010 & 16.3 & $\mathrm{~F}$ & E-012 & NA \\
\hline E-014 & 14.3 & $\mathrm{M}$ & E-013 & NA \\
\hline E-016 & 12.2 & $\mathrm{~F}$ & E-015 & NA \\
\hline E-017 & 11.2 & $\mathrm{~F}$ & E-018 & NA \\
\hline E-022 & 7.3 & $\mathrm{~F}$ & E-021 & NA \\
\hline E-029 & 14.5 & M & E-030 & NA \\
\hline
\end{tabular}

FAP: Familial adenomatous polyposis; PID: participant identification number.

age $=12.8$ years $(\mathrm{SD}=4.0)$, age range $=6.1-16.9$ years $]$ and 9 siblings to serve as controls [ 4 male, average age $=12.2$ years $(\mathrm{SD}=3.3)$, age range $=7.3-16.3$ years]. None of the participants reported history of known hearing loss. Children with FAP were divided into three main categories based on genotype as previously described (attenuated, classic and severe) (15). Subject demographics are found in Table I.

Procedures. Hearing evaluations followed nationally established clinical standards for ototoxic monitoring (16). Hearing evaluations included the following standard procedures performed by licensed audiologists: otoscopy, tympanometry, ipsilateral and contralateral acoustic reflex threshold testing, pure-tone audiometry including the extended high-frequency range (250-20,000 Hz), speech perception audiometry, and distortion product otoacoustic emission testing.

Otoscopic examination was completed to evaluate the health of the outer ear and tympanic membrane, and to ensure cerumen 
Findlen et al: Hearing in Pediatric FAP

Table II. Summary of audiometric data including pure tone averages, high-frequency pure tone averages, tympanometry, speech recognition scores, and otoacoustic emissions, for familial adenomatous polyposis (FAP) and non-FAP participants.

\begin{tabular}{|c|c|c|c|c|}
\hline \multirow[t]{2}{*}{ Audiometric measure } & \multicolumn{2}{|c|}{ FAP participants } & \multicolumn{2}{|c|}{ Non-FAP participants } \\
\hline & Right ear & Left ear & Right ear & Left ear \\
\hline \multicolumn{5}{|c|}{ Pure-tone averages, Mean (SD) } \\
\hline Standard PTA & $8.4(5.9)$ & $9.8(8.9)$ & $7.0(3.5)$ & $7.2(4.8)$ \\
\hline High-frequency PTA & $4.6(17.6)$ & $5.2(11.6)$ & $-4.6(11.9)$ & $-2.9(12.2)$ \\
\hline \multicolumn{5}{|l|}{ Speech recognition } \\
\hline \% Correct, Mean (SD) & $97.3(4.2)$ & $96.2(5.3)$ & $96.0(4.7)$ & $94.9(4.1)$ \\
\hline Range (\%) & $86-100$ & $80-100$ & $90-100$ & $90-100$ \\
\hline \multicolumn{5}{|l|}{ Tympanometry, \% (n) } \\
\hline Normal & $85(17)$ & $85(17)$ & $100(9)$ & $100(9)$ \\
\hline Abnormal & $15(3)$ & $15(3)$ & $0(0)$ & $0(0)$ \\
\hline \multicolumn{5}{|c|}{ Otoacoustic emissions, \% (n) } \\
\hline Present & $65(13)$ & $50(10)$ & $22(2)$ & $33(3)$ \\
\hline Partially present & $35(7)$ & $50(10)$ & $78(7)$ & $67(6)$ \\
\hline
\end{tabular}

occlusion would not negatively affect results of remaining testing procedures. If cerumen was identified as more than partially occluding, brief attempts were made to clear the ear manually. Tympanometry was performed to measure peak compensated static acoustic admittance measures using a $226 \mathrm{~Hz}$ probe tone (GrasonStadler Instruments Middle Ear Analyzer Models TympStar and TympStar Pro, Eden Prairie, MN, USA) calibrated annually to ANSI standard 3.39-R2002. Tympanometry metrics of peak static compliance and middle ear pressure were compared to normative data to evaluate the status of the middle ear (17). Ipsilateral and contralateral acoustic reflex testing was completed via standard clinical protocol using a $226 \mathrm{~Hz}$ probe tone and stimulation tones of $500 \mathrm{~Hz}, 1,000 \mathrm{~Hz}$, and $2,000 \mathrm{~Hz}$. Reflexes were considered present when elicited at $95 \mathrm{~dB}$ or less, elevated when elicited at 100 $\mathrm{dB}$ or more, and absent if not elicited at the limits of the equipment.

Pure tone audiometry was performed in a double walled sound booth (Industrial Acoustics Company, North Aurora, IL, USA) calibrated re: ANSI S3.1-R2003. Pure tone thresholds were assessed with an audiometer (Grason-Stadler Instruments Clinical Audiometers 61 and AudioStar Pro) calibrated annually re: ANSI S3.6-R2004. Insert earphones (Etymotic Research ER 3A, Elk Grove Village, IL, USA) were used for air conduction pure tone threshold testing from 250-8000 Hz. Supra-aural headphones (Sennheiser HDA 200, Wedemark, Germany) were used for air conduction pure tones above $8000 \mathrm{~Hz}$. A Radioear (B71 or B81, New Eagle, PA, USA) transducer was used for bone conduction pure tones testing. Following clinical standards, threshold search was completed using a Carhart-Jerger Modified Hughson-Westlake procedure (18). Participants responded to pure tone presentations by pushing the patient response button or raising their hand each time they heard a tone. Standard pure-tone average (PTA) was calculated using the mean of $500 \mathrm{~Hz}, 1000 \mathrm{~Hz}$, and $2000 \mathrm{~Hz}$ responses for each ear. High-frequency pure-tone average (HFPTA) was calculated for each participant using the mean of $10,000 \mathrm{~Hz}, 12,000$ $\mathrm{Hz}, 14,000 \mathrm{~Hz}$, and 16,000 $\mathrm{Hz}$ per previous literature (19).

Speech Audiometry followed pure-tone testing and included both speech reception thresholds and speech perception testing. Speech thresholds were obtained with a simple bracketing procedure until three of five words were identified correctly denoting threshold. Speech perception testing was completed using recorded Central Institute for the Deaf (CID-W22) word lists (Auditec, St. Louis, MO, USA) presented at $40 \mathrm{~dB}$ SL re: standard PTA via the audiometer. Lastly, distortion product otoacoustic emissions (DPOAE) were recorded using the Biologic Scout clinical otoacoustic emissions machine (Natus, San Carlos, CA, USA) using an ototoxic monitoring protocol measuring emissions between 1500 $\mathrm{Hz}$ and 10,000 Hz. DPOAEs at specific frequencies were deemed present when absolute response amplitude was greater than $-6 \mathrm{~dB}$ and DPOAE-to-noise ratio was at least $6.0 \mathrm{~dB}$.

For statistical analysis, two-sample unpaired t-tests with unequal variances were performed to test for differences between individuals with FAP and those without FAP in PTA and HFPTA. All statistical tests were two-sided and results are reported at their nominal levels. $p$-values $<0.05$ were considered statistically significant. All analyses were performed in $\mathrm{R}$ version 4.0.

\section{Results}

All but two subjects (E-001 and E-031, both with FAP) had normal audiologic evaluation results as measured by pure tone audiometry and speech audiometry (Table II). All subjects except for E-031 presented with symmetric hearing (mean PTA difference between ears=3.7) and therefore graphical depiction of audiometric data represent the right ear only (Figure 1). All subjects with normal hearing presented with mean speech recognition scores of $96 \%$ and $97 \%$ correct for the right and left ears, respectively, with a range from $88 \%$ to $100 \%$ correct. Tympanometry results were within normal limits for compliance and peak pressure for all non-FAP participants and the majority $(75 \%, n=15)$ of the participants with FAP. Of the five FAP participants who had abnormal tympanometry, three $(60 \%)$ had a history of 


\section{Hearing thresholds}

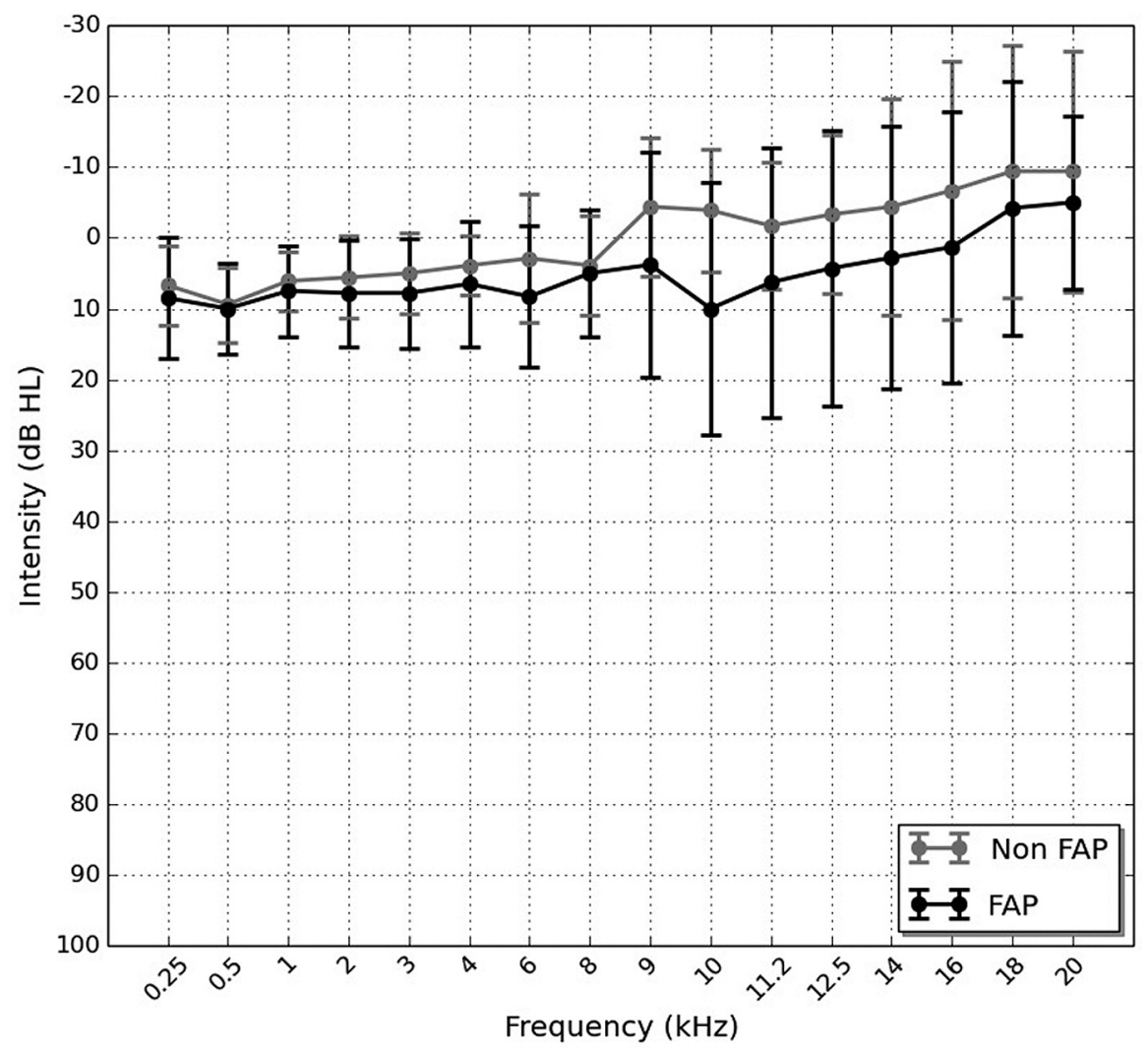

Figure 1. Mean right ear audiometric thresholds for FAP (n=20) and Non-FAP (n=9) subjects from $250 \mathrm{~Hz}$ through 20,000 Hz. Bars represent one standard deviation from the mean. FAP: Familial adenomatous polyposis.

otitis/effusion prompting bilateral tube placement in early childhood per participant/family report. All participants presented with either completely present or partially present DPOAEs. Two of the FAP participants who presented with partially present DPOAEs also had abnormal tympanometry on the day of testing, while the two additional patients were those who presented with hearing loss. The remaining participants who presented with partially present DPOAEs had no other abnormal test results, and therefore missing responses may reflect individual differences at select frequencies or probe placement impacting measurement.

One participant (E-001) presented with mild sensorineural hearing loss bilaterally (PTA right ear=25.0, left ear=26.7) and had speech recognition scores of $80 \%$ and $86 \%$ for the right and left ears, respectively. Etiology of the hearing loss is unknown at this time; however, no family history of hearing loss was reported. One other participant (E-031) presented with mild to moderate low-frequency conductive hearing loss bilaterally and right ear hearing that was significantly better than left ear (PTA right ear=20.0, left ear=36.7). Speech recognition was $100 \%$ for both ears. Otologic history was notable for previous external auditory canal cyst removal from the right ear prior to two years of age, otherwise no other otologic symptoms were present. On the day of testing, tympanometry revealed hypermobility for the right ear but mobility was normal for the left ear. There was no family history of hearing loss and no previous concerns regarding hearing. Both participants were referred to ear, nose, and throat physicians for further assessment and management. Of note, these hearing tests were the first ever undergone for both of these patients at ages 16.4 and 17.0 years, respectively. Therefore, onset and/or progression of hearing loss is unknown due to the lack of previous audiometric testing.

Results of the statistical analysis revealed no statistically significant differences between those who had FAP $v s$. those without FAP in this sample for right ear PTA $(p=0.44)$, left ear PTA $(p=0.31)$, right ear HFPTA $(p=0.12)$ or left ear HFPTA $(p=0.16)$. Additionally, no clinically significant differences were observed between groups. 


\section{Discussion}

Any chemopreventative agent used in the pediatric population must present little or no risk to the patient while offering a clear benefit. This is particularly important for otherwise healthy children and adolescents who will likely receive treatment over decades with the goal of minimizing adult morbidity. Since the $A P C$ gene/protein plays a role in hearing and cochlear development (2), the specific aim of the current study was to establish the baseline hearing status of children and adolescents with genotype positive FAP and compare results to a group of genotype negative/unaffected siblings. The inclusion of a sibling control group allows for direct comparison of pediatric FAP cohort to a group of children with both genetic and environmental similarities. This is a novel approach in evaluating hearing in this population given that previous studies compared hearing thresholds to age-normative values.

All but two participants presented with normal audiometric findings. Both hearing impaired participants were in the FAP cohort, however, the etiology of hearing loss was either unknown or potentially due to an unrelated otologic issue. Given that one participant presented with conductive hearing loss with site of lesion traced to the middle ear system as opposed to the cochlea, it is highly unlikely that the etiology was related to FAP and the APC gene mutation. In contrast to observations in the adult population, this study (although small in size) suggests that genotype positive FAP children/adolescents are not predisposed to hearing loss as a function of their disease. These are promising data in terms of the potential use of DFMO in FAP children but reinforces the need for careful baseline and ongoing audiometric monitoring during clinical trials that use potentially ototoxic chemopreventative agents.

There are two main limitations of this study, specifically the limited number of participants and the limited number of siblings available to serve as a control cohort. Given the rarity of FAP, it is not surprising that this pediatric study has a relatively small sample size. Additionally, the inclusion of siblings as a control cohort was planned due to the absence of studies in this population that have used a valid (i.e. familial) control group. Recruitment for this study was quite rigorous, however, the number of available siblings who fit the age criteria were limited as many parents were unwilling to remove siblings from school for testing. Overall, the control cohort was helpful in understanding that despite pathophysiology of the APC gene and FAP in general, no significant differences in hearing exist between those with FAP and an ecologically valid control group.

\section{Conclusion}

The prevalence of hearing loss in children with FAP is very low and no differences were observed between the hearing thresholds of FAP patients and their siblings. This suggests that the presence of the APC mutation is not necessarily indicative of higher risk for baseline hearing loss. Future clinical trials in the chemopreventative management of FAP should include audiometric monitoring to evaluate the baseline presence of hearing loss and any potential effects of therapeutic drugs.

\section{Conflicts of Interest}

The Authors have no conflicts of interest to report regarding this study.

\section{Authors' Contributions}

Ursula M. Findlen- collected audiometric data, conceptualized the analysis, and wrote drafts of the manuscript. Rebecca L. Beltconceptualized and collected the audiometric data, provided substantive edits to the manuscript. Ling Fan- consented subjects, coordinated scheduling, collected samples for genetic testing, and provided substantive edits to the manuscript. Nikia Bridgescollected audiometric data and provided substantive edits to the manuscript. Jason Benedict- provided statistical analysis of the study results and manuscript narrative pertaining to the analysis. Steven H. Erdman- conceptualized the study design, enrolled subjects, analyzed genetic data, and provided substantive edits to the manuscript.

\section{Acknowledgements}

This study was funded through an unrestricted grant from Cancer Prevention Pharmaceuticals, Inc. The authors would like to acknowledge Dr. Kirstin Chiasson for her contribution to the study design at the onset of this project.

\section{References}

1 Galiatsatos P and Foulkes WD: Familial adenomatous polyposis. Am J Gastroenterol 101(2): 385-398, 2006. PMID: 16454848. DOI: $10.1111 / \mathrm{j} .1572-0241.2006 .00375 . x$

2 Patuzzi R: Cochlear micromechanics and macromechanics. Springer, New York, NY, pp. 186-257, 1996.

3 Hickman TT, Liberman MC and Jacob MH: Adenomatous polyposis coli protein deletion in efferent olivocochlear neurons perturbs afferent synaptic maturation and reduces the dynamic range of hearing. J Neurosci 35(24): 9236-9245, 2015. PMID: 26085645. DOI: 10.1523/JNEUROSCI.4384-14.2015

4 Jones SE, Joice P, Cochrane L, Thoresen I, Quyn A and Nähke I: Is familial adenomatous polyposis associated with sensorineural hearing loss? Int J Audiol 49(10): 762-764, 2010. PMID: 20874049. DOI: 10.3109/14992027.2010.491098

5 Lutz T, Lopez R, Burke C, Lynch P, Hawk E, Griebel D, Phillips $\mathrm{R}$, Latchford A and Hennie H: Is Hearing loss an extracolonic manifestation of FAP? American Journal of Gastroenterology 101: S554, 2020. DOI: 10.14309/00000434-200609001-01456

6 Barry EL, Baron JA, Bhat S, Grau MV, Burke CA, Sandler RS, Ahnen DJ, Haile RW and O'Brien TG: Ornithine decarboxylase polymorphism modification of response to aspirin treatment for 
colorectal adenoma prevention. J Natl Cancer Inst 98(20): 14941500, 2006. PMID: 17047198. DOI: 10.1093/jnci/djj398

7 Burke CA, Dekker E, Lynch P, Samadder NJ, Balaguer F, Hüneburg R, Burn J, Castells A, Gallinger S, Lim R, Stoffel EM, Gupta S, Henderson A, Kallenberg FG, Kanth P, Roos VH, Ginsberg GG, Sinicrope FA, Strassburg CP, Van Cutsem E, Church J, Lalloo F, Willingham FF, Wise PE, Grady WM, Ford M, Weiss JM, Gryfe R, Rustgi AK, Syngal S and Cohen A: Eflornithine plus sulindac for prevention of progression in familial adenomatous polyposis. N Engl J Med 383(11): 10281039, 2020. PMID: 32905675. DOI: 10.1056/NEJMoa1916063

8 Lynch PM, Burke CA, Phillips R, Morris JS, Slack R, Wang X, Liu J, Patterson S, Sinicrope FA, Rodriguez-Bigas MA, Half E, Bulow S, Latchford A, Clark S, Ross WA, Malone B, Hasson H, Richmond $\mathrm{E}$ and Hawk E: An international randomised trial of celecoxib versus celecoxib plus difluoromethylornithine in patients with familial adenomatous polyposis. Gut 65(2): 286295, 2016. PMID: 25792707. DOI: 10.1136/gutjnl-2014-307235

9 McLaren CE, Fujikawa-Brooks S, Chen WP, Gillen DL, Pelot D, Gerner EW and Meyskens FL Jr: Longitudinal assessment of air conduction audiograms in a phase III clinical trial of difluoromethylornithine and sulindac for prevention of sporadic colorectal adenomas. Cancer Prev Res (Phila) 1(7): 514-521, 2008. PMID: 19139001. DOI: 10.1158/1940-6207.CAPR-080074

10 Meyskens FL Jr, McLaren CE, Pelot D, Fujikawa-Brooks S, Carpenter PM, Hawk E, Kelloff G, Lawson MJ, Kidao J, McCracken J, Albers CG, Ahnen DJ, Turgeon DK, Goldschmid $\mathrm{S}$, Lance $\mathrm{P}$, Hagedorn $\mathrm{CH}$, Gillen DL and Gerner EW: Difluoromethylornithine plus sulindac for the prevention of sporadic colorectal adenomas: a randomized placebo-controlled, double-blind trial. Cancer Prev Res (Phila) 1(1): 32-38, 2008. PMID: 18841250. DOI: 10.1158/1940-6207.CAPR-08-0042

11 Doyle KJ, McLaren CE, Shanks JE, Galus CM and Meyskens FL: Effects of difluoromethylornithine chemoprevention on audiometry thresholds and otoacoustic emissions. Arch Otolaryngol Head Neck Surg 127(5): 553-558, 2001. PMID: 11346432. DOI: 10.1001/archotol.127.5.553
12 Nie L, Feng W, Diaz R, Gratton MA, Doyle KJ and Yamoah EN: Functional consequences of polyamine synthesis inhibition by L-alpha-difluoromethylornithine (DFMO): cellular mechanisms for DFMO-mediated ototoxicity. J Biol Chem 280(15): 15097 15102, 2005. PMID: 15718247. DOI: 10.1074/jbc.M409856200

13 McWilliams ML, Chen GD and Fechter LD: Characterization of the ototoxicity of difluoromethylornithine and its enantiomers. Toxicol Sci 56(1): 124-132, 2000. PMID: 10869460. DOI: 10.1093/toxsci/56.1.124

14 Croghan MK, Aickin MG and Meyskens FL: Dose-related alpha-difluoromethylornithine ototoxicity. Am J Clin Oncol 14(4): 331-335, 1991. PMID: 1862765. DOI: 10.1097/000 00421-199108000-00012

15 Nieuwenhuis MH and Vasen HF: Correlations between mutation site in APC and phenotype of familial adenomatous polyposis (FAP): a review of the literature. Crit Rev Oncol Hematol 61(2): 153-161, 2007. PMID: 17064931. DOI: 10.1016/j.critrevonc. 2006.07.004

16 Guidelines for manual pure-tone threshold audiometry. ASHA 20(4): 297-301, 1978. PMID: 656172.

17 Margolis R and Heller J: Screening Tympanometry: Criteria for Medical Referral. International Journal of Audiology 26(4): 197208, 2011. DOI: 10.3109/00206098709081549

18 Carhart R and Jerger J: Preferred method for clinical determination of pure-tone thresholds. Journal of Speech and Hearing Disorders 24(4): 330-345, 2019. DOI: 10.1044/jshd.2404.330

19 Le Prell CG, Spankovich C, Lobariñas E and Griffiths SK: Extended high-frequency thresholds in college students: effects of music player use and other recreational noise. J Am Acad Audiol 24(8): 725-739, 2013. PMID: 24131608. DOI: 10.3766/jaaa.24.8.9 